\title{
A Novel Multi-focus Image Fusion Method using Pulse Coupled Neural Network in Nonsubsampled Contourlet Transform Domain
}

\author{
Chaoben $\mathrm{Du}^{1,2}$, Haifeng $\mathrm{Yan}^{1}$, Shesheng $\mathrm{Gao}^{1}$ and Gaoge $\mathrm{Hu}^{1}$ \\ ${ }^{1}$ School of Automatic, Northwestern Polytechnical University,Xi'an, 710072 China \\ ${ }^{2}$ State Grid Jinchang power supply company,Jinchang,737100, China \\ dcbxjdaxue@163.com
}

\begin{abstract}
Keywords: multi-focus image fusion, nonsubsampled contourlet transform, pulse coupled neural network, edge feature.
\end{abstract}

\begin{abstract}
In this paper, a novel image fusion method is proposed which combines nonsubsampled contourlet transform (NSCT) with PCNN. Firstly, it makes use of the NSCT's shift invariance to suppress the pseudo-Gibbs phenomena around singularities, which appears in the DWT. Secondly, the edge feature is used to motive the improved PCNN model, to retain more edge and texture details. Some experiments are performed in images such as clock, pepsi and book images comparing the proposed algorithm with the SML-CT,PCNN-NSCT and SF-NSCT-PCNN methods The experimental results show that the proposed algorithm can not only extract more important visual information from source images, but also effectively avoid the introduction of artificial information.
\end{abstract}

\section{INTRODUCTION}

Multi-focus image fusion is one part of the image fusion domain, which is still concerned so far. Recently, multi-resolution analysis is widely used in image fusion, which includes wavelets, Laplacian pyramid and gradient pyramid, the ratio of low pass pyramid and contrast pyramid. The traditional wavelet transform can capture the limited direction information, but cannot capture the outline information and cause pseudo-Gibbs phenomena around singularities. To solve these issues, some researchers put forward stationary contourlet transform [1] and àtrous wavelet transform [2], but edge information are missed. Besides, the nonsubsampled contourlet transform (NSCT) put forward by Cunha also can solve these problems with its shift invariance, anisotropy and rich directions [3].

Pulse coupled neural network (PCNN) is proposed by Eckhorn in 1900, which owns some excellent characters, such as global coupling and pulse synchronization [4].Reference [5] presents a multifocus image fusion method in Sharp Frequency Localized Contourlet Transform(SFLCT) domain based on a sum-modified-Laplacian. In Ref. [6] proposed image fusion algorithm based on nonsubsampled contourlet transform and pulse coupled neural netwrork. By using NSCT,the input images were decomposed into a number of sub-images with various scales and directional features.Then,based on PCNN,a fusion rule is given.Reference [7] presents Image Fusion Algorithm Based on Spatial Frequency-Motivated Pulse Coupled Neural Networks in Nonsubsampled Contourlet Transform Domain .in which NSCT is associated with PCNN and used in image fusion to make full use of the characteristics of them.Spatial frequency in NSCT domain is input to motivate PCNN and coeffcients in NSCT domain with large firing times are selected as coeffcients of the fused image.

In this paper,we introduce the energy of edge (EOE) to stimulate the PCNN, which design a fusion method based on modified PCNN and NSCT which introduces energy of edge. It makes use of the NSCT's shift invariance to suppress the pseudo-Gibbs phenomena around singularities, which appears in the DWT. Then, the edge feature is used to motive the improved PCNN model, to retain more edge and texture details. 


\section{MODIFIED PULSE COUPLED NEURAL NETWORK}

The traditional PCNN is a feedback network and one neuron of PCNN consists of three parts: the receptive parts, the modulation field, and the pulse generator.

By the analyzing the structure of neuron, there are many parameters to determine, which is not beneficial to practical application. Some researchers propose the simplified PCNN model according to the different goal. However, this does not get the ideal results in image fusion. So, a modified PCNN model is adopted in this paper. Besides, the linking coefficient $\beta$ and external stimulus play a key role in PCNN, which directly decide the performance of PCNN. In the literature [4], the author points out that the $U$ will produce the false decisions while linking with low-intensity pixel of the image that just below the threshold potential $\theta$. So, he proposes the Sigmoid function for processing of input quality. Sigmoid response of output is used as a feed back to the $\mathrm{L}$ channel. So we get the mathematical equations of Modified PCNN are shown from (1) to (7)

$$
\begin{aligned}
& F_{i, j}=S_{i, j} \\
& L_{i, j}[n]=\sum_{k, l \in D} W_{i, j, k, l}\left(Y_{k, l}[n-1] X_{i, j}[n-1]\right)+\exp \left(-\alpha_{L}\right) L_{i, j}[n-1] \\
& U_{i, j}[n]=F_{i, j}[n]\left(\alpha_{U}+\beta_{i, j} L_{i, j}[n]\right) \\
& \theta_{i, j}[n]=L_{i, j} V_{\theta} Y[n]+\exp \left(-\alpha_{\theta}\right) \theta_{i, j}[n-1] \\
& Y_{i, j}[n]=\operatorname{step}\left(U_{i, j}[n]-\theta_{i, j}[n]\right) \\
& X_{i, j}[n]=1 /\left(1+\exp \left(-2\left(U_{i, j}-\theta_{i, j}\right)\right)\right. \\
& T_{i, j}[n]=T_{i, j}[n-1]+Y_{i, j}[n]
\end{aligned}
$$

Where $\mathrm{i}$ and $\mathrm{j}$ denote the pixel space coordinate, $\mathrm{D}$ denotes the neighborhood around the pixel at position $(\mathrm{i}, \mathrm{j}), \mathrm{m}$ and $\mathrm{n}$ are the decomposed scale and direction, $\alpha_{\mathrm{U}}$ is the weighted factor, $\alpha_{\mathrm{L}}$ and $\alpha$ ${ }_{\theta}$ are the delay constants, $\mathrm{W}_{\mathrm{i}, \mathrm{j}, \mathrm{k}, \mathrm{l}}$ is the synaptic weight $\mathrm{V}_{\mathrm{L}}$ and $\mathrm{V}_{\theta}$ are the amplitude gain, $\mathrm{n}$ denotes the iteration number currently, step is the step response, when $U_{i, j}$ exceeds $\theta_{i, j}$, it will output 1 ,otherwise it will output 0 . The linking coefficient $1>\beta>0$ is a key role in PCNN which decides the lifting range and exciting character in the region mentioned in some literatures. In general, the weighing coefficient is a constant, which is a limitation for the adaptation of the method. So, some measures are made to strength the ability to adaption. Because the human eyes is more sensitive to region than the signal pixel .To make use of the surrounding information, The size of the region around $S_{i, j}$ is chosen as $(2 N+1) \times(2 M+1)$. Hence the region feature $D_{i, j}$ is computed using equations(8)

$$
D_{i, j}=\sum_{k=-N}^{N} \sum_{-M}^{M} S_{i, j}
$$

At last, the Sigmoid activation function is used to calculate the linking coefficient $\beta_{\mathrm{i}, \mathrm{j}}$ as shown in equations(9), Where, $\mathrm{k}$ is the weighing constant which is assigned by experiment or experience.

$$
\beta_{i, j}=\frac{2}{\left(1+\exp \left(-\kappa D_{i, j}\right)\right)}-1
$$

\section{IMAGE FUSION METHOD BASE ON EOE-PCNN-NSCT}

\subsection{Energy of edge}

The original image fusion method based on PCNN uses the gray value of pixel as the external stimulus which is representing the feature of image. Recently, a new image fusion method combines NSCT and SF is proposed,which get better result in fused image. Moreover, the author points out that the SML or EOL is utilized as the external input of PCNN, which would improve the method performance. We can know that human eye is sensitive to edge information. The EOL can better denote the edge feature than EOL,SF,SML. So we choose the EOE as the external stimulus of PCNN in NSCT domain, which is computed by equation(10). 


$$
S_{i, j}=\sum_{k, l \in D} \omega(k, l) L E(k, l)
$$

Where the $\mathrm{w}(\mathrm{k}, \mathrm{l})$ is weighted factor where the sum of its elements equals to 1 , $\mathrm{D}$ is the neighborhood window around position (i, j), LE (k, l) is calculated using equation(11)

$$
L E(i, j)=(E 1 * Z(i, j))^{2}+(E 2 * Z(i, j))^{2}+(E 3 * Z(i, j))^{2}
$$

Where $\mathrm{Z}(i, \mathrm{j})$ is the coefficient in NSCT domain, E1, E2, E3 denote the directional filtering operators, which are shown as follows

$$
E 1=\left|\begin{array}{ccc}
-1 & -1 & -1 \\
2 & 2 & 2 \\
-1 & -1 & -1
\end{array}\right| \quad E 2=\left|\begin{array}{ccc}
-1 & 2 & -1 \\
-1 & 2 & -1 \\
-1 & 2 & -1
\end{array}\right| \quad E 3=\left|\begin{array}{ccc}
-1 & 0 & -1 \\
0 & 4 & 0 \\
-1 & 0 & -1
\end{array}\right|
$$

So the edge feature can be extracted by three filtering operators defined above [8].

\subsection{The EOE-PCNN-NSCT-based image fusion method}

According to the discussion above, we get fusion method of this paper. In other image fusion algorithm, the firing times is regarded as the criteria for selecting the fusion coefficient. The larger firing times corresponding to the position is choose. So we get the flowchart of the proposed image fusion method is shown in Fig.1.

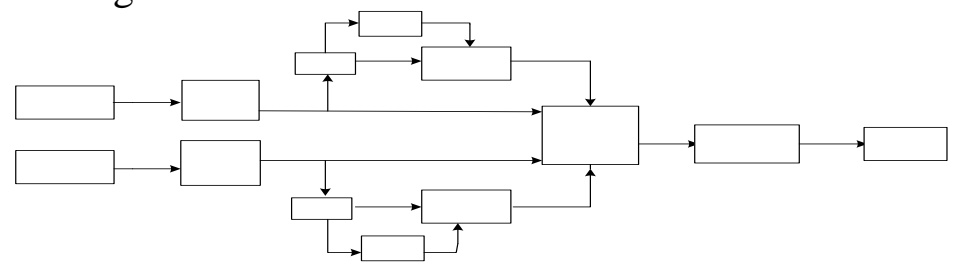

Fig.1. The flowchart of EOE-PCNN-NSCT-based image fusion

Supposed the source images have been registered, the fusion method can be conducted on them. The process of the image fusion algorithm is as follows.

1) Decompose the source image A and B using NSCT.

2) Calculate the EOE use Eq. and get the feature matrix $S^{A}{ }_{i, j}$ and $S^{B}{ }_{i, j}$.

3) Select the fused coefficient via PCNN and the fusion rule.

a. Initialize the PCNN parameters, $L_{i, j}=0, \theta_{i, j}=0, Y_{i, j}=0 . T_{i, j}=0$.

b. Calculate the linking strength $\beta$ using Eq. (8) and Eq. (9).

c. Get the firing times matrix $\mathrm{T}^{\mathrm{A}}{ }_{\mathrm{i} \text { j }}$ and $\mathrm{T}^{\mathrm{A}}{ }_{\mathrm{i}, \mathrm{j}}$ according to Eq.(1) to Eq.(7), until the iteration number $\mathrm{n}=\mathrm{N}_{\max }, \mathrm{N}_{\max }$ is the max iteration times.

d. The fused coefficient is F (i, j) selected by Eq. (12).

$$
Z^{F}(i, j)= \begin{cases}Z^{A}(i, j) & \text { if }: L T S^{A} \geq L T S^{B} \\ Z^{B}(i, j) & \text { if }: L T S^{A}<L T S^{B}\end{cases}
$$

Where LTS $^{\mathrm{A}}$ and $\mathrm{LTS}^{\mathrm{B}}$ is the sum of local firing times, is computed by Eq. (13).

$$
\operatorname{LTS}(i, j)=\sum_{k, l \in D} T(i, j)
$$

4)Get the fused image via reconstructed the fused coefficient $Z^{F}(i, j)$.

\section{THE EXPERIMENTAL RESULTS AND ANALYSIS}

To test the performance of the proposed fusion method, several experiments are conducted on the proposed methods. Three group images are selected for testing namely the clock, pesis and book which are shown in Fig.3. 


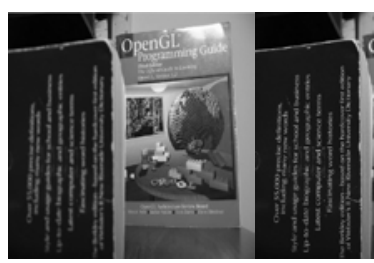

(a) (b)

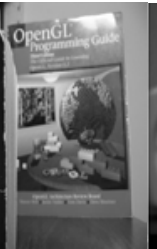

(b)

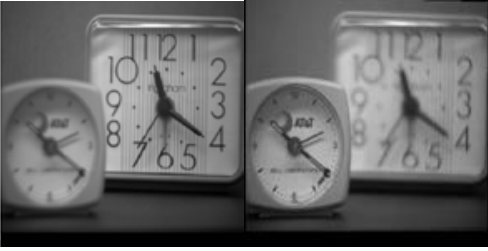

(c)

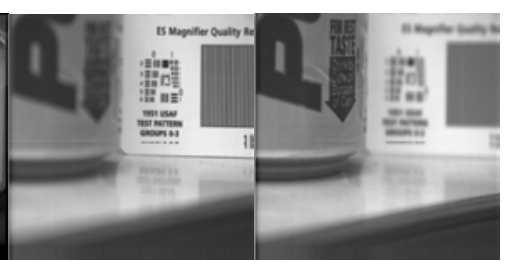

(e) (f)

Fig.3. Source for testing : (a) and (b) are right and left focus images of book, (c) and (d) are right and left focus images of clock, (e) and (f) are right and left focus images of pepsi.

The parameters of PCNN used in the experiments are listed as follows: $\alpha_{U}=0.7298, \alpha_{L}=0.2, \alpha$

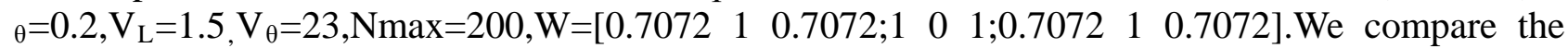
proposed algorithm with a number of the state-of- the-art algorithms, such as Sum-modified-Laplacian contourlet (SML-CT), PCNN-NSCT, the spatial frequency motivated PCNN (SF-PCNN-NSCT) [6]. Subjective criteria and objective criteria are used to evaluate the performance of the proposed method in the experiments.

\subsection{Subjective evaluation}

The book is selected for the subjective testing. The results of the different fusion methods are shown in Fig.3 (d)-(f).

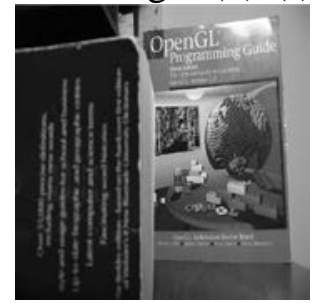

(a)

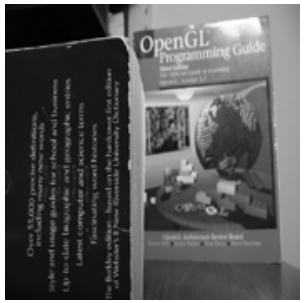

(b)

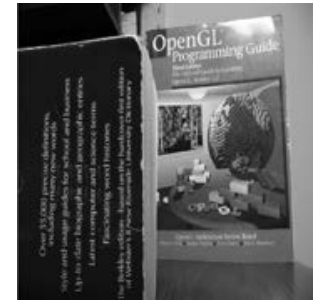

(c)

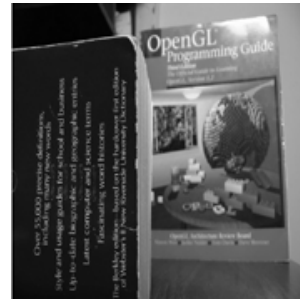

(d)

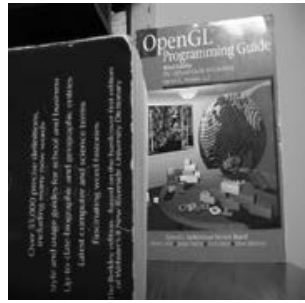

(e)

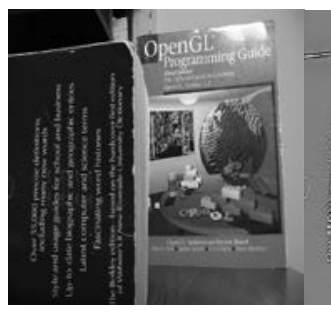

(f) (g)

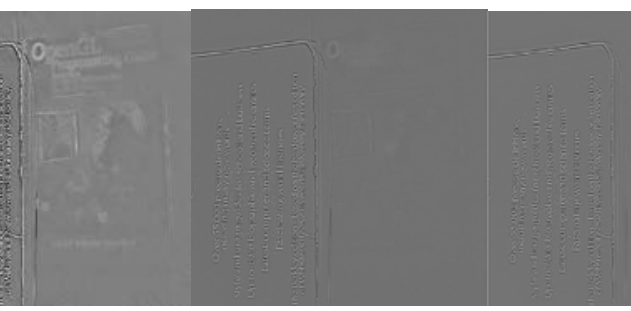

(h) (i) (j)

Fig.4. Source images and fused images of different fusion method: (a) focus on the left ,(b)focus on the right,(c)fused image using SML-CT ,(d)fused image using PCNN-NSCT, (e)fused image using

SF-PCNN-NSCT,(f)fused image by the proposed method (g)difference between (c) and (a), (h) difference between (d) and (a), (i) difference between (e) and (a),(j)difference between (f) and (a).

We use the fused images subtract the left focus image and get the difference images are shown Fig.4 (g)-(i).The difference between (c) and (a), or (d) and a) in right parts is obvious. This means the information of the original is not better transformed to the fused image. However, the difference between (e) and (a), or (f) and (a) is not obvious. This indicates that the original information almost is transformed into the fused image. And that the difference between SF-PCNN-NSCT and EOE-PCNN-NSCT is still to distinguish. So we evaluate them in objective evaluation part. Besides, the proposed method gets better effect than MAX-NSCT and PCNN-NSCT.

\subsection{Objective evaluation}

1) Mutual Information

Mutual information (MI) is the measure of correlation between two random variables, or a random variable contains information that contains a measure of another random variable. The larger value of the mutual information, the more information extracted from the original image [9]. The total mutual information can be computed using the following equations

$$
\mathrm{MI}_{\mathrm{T}}=\mathrm{MI}_{\mathrm{FA}}+\mathrm{MI}_{\mathrm{FB}}
$$

Where $M I_{F A}$ or $M I_{F B}$ is denotes the mutual information between the Fused image and original image, which is defined as follows 


$$
M I_{F A}=\sum_{i=0}^{M-1} \sum_{j=0}^{N-1} p_{F A}(i, j) \log \frac{p_{F A(i, j)}}{p_{F}(i) p_{A}(j)}
$$

2) $Q^{A B / F}$

$\mathrm{Q}^{\mathrm{AB} / \mathrm{F}}$ denotes the retaining amount of the edge information of the source image which using the Sobel edge detection operator. The larger the value is, the more edge information is transformed to the fused image [10]. All the three group images are used to testify the objective performance in this paper. We compare the proposed fusion algorithm with the other methods. The different fused results of different fusion methods are shown in Table.1.

\begin{tabular}{cccccc}
\multicolumn{6}{c}{ Table.1 Fusion results of the four methods in MI and $\mathrm{Q}^{\mathrm{AB} / \mathrm{F}}$} \\
\hline Image & criteria & SML-CT & PCNN-NSCT & SF-NSCT-PCNN & Proposed method \\
\hline clock & $\mathrm{MI}$ & 6.5603 & 6.8186 & 7.4051 & 0.3949 \\
& $\mathrm{Q}^{\mathrm{AB} / \mathrm{F}}$ & 0.6672 & 0.6826 & 7.6863 & 0.7241 \\
pepsi & $\mathrm{MI}$ & 6.6514 & 6.9738 & 0.7323 & 8.2731 \\
& $\mathrm{Q}^{\mathrm{AB} / \mathrm{F}}$ & 0.7636 & 0.7738 & 7.9767 & 0.7865 \\
book & $\mathrm{MI}$ & 7.0281 & 7.4666 & 0.6893 & 8.9886 \\
& $\mathrm{Q}^{\mathrm{AB} / \mathrm{F}}$ & 0.6968 & 0.6794 & 0.7217 \\
\hline
\end{tabular}

We can learn that the proposed image fusion algorithm gets better than the other algorithms in $\mathrm{MI}$ and $\mathrm{Q}^{\mathrm{AB} / \mathrm{F}}$. It can transfer more information form source image to the fused image whiling retaining more edge information. In the subjective evaluation, it is hard to distinguish the proposed method and the SF-PCNN-NSCT. But, the proposed method outperforms the SF-PCNN-NSCT in objective evaluation.

\section{Conclusion}

The traditional PCNN model has many parameters to be set, which is not good for image processing. The traditional model is modified in this paper to simplify the complexity. The linking strength $\beta$ of the modified PCNN model is adjusted according to EOE by Sigmoid response function. The EOE is extracted from the NSCT domain to motivate the modified PCNN which. The proposed method is evaluated in subjective and objective criteria. Besides, the proposed method is compared with other methods so as to illustrate the superiority of the proposed. The experimental results of the proposed methods get better results than the other methods in $\mathrm{MI}$ and QAB/F.

\section{Acknowledgement}

In this paper, the research was sponsored by the Aerospace Science and Technology Innovation Foundation of China Aerospace Science and Technology Corporation (Project Number: CASC201102)

\section{References}

[1] Dexiang ZHANG, Xiaopei WU, Qingwei GAO , Xiao-jing GUO ,’Fusion of Polarimetric SAR Image Based on Stationary Contourlet Transform”, Journal of University of Electronic Science and Technology of China, vol.39,no.2,pp.200-204,2010.

[2]M.J. Choi , "A new intensity-hue-saturation fusion approach to image fusion with a tradeoff parameter,” IEEE Trans. Geosci. Remote Sens., vol.44, no.6, pp.1672-1682,2006.

[3]Cunha A L da, Jianping Zhou, Do M N, “The nonsubsampled Contourlet transform: Theory, design and application, "IEEE Transactions On Image Processing, vol. 15,no.10,pp.3089-3101,2006.

[4]R. Eckhorn, H.J. Reiboeck, M. Arndt, P.W. Dicke, Feature linking via synchronization among distributed assemblies: Simulation of results from cat cortex, Neural computation, pp.293-307, 1900.

[5] QU Xiao-bo, YAN Jing-wen,YANG Gui-de , "Multifocus image fusion method of sharp frequency localized Contourlet transform domain based on sum-modified-Laplacian”. Optics and 
Precision Engineering, Vol.17 ,no.5,pp.1204-1213, 2009.

[6] XLAO Wei, WANG Rong-feng,“Image fusion algorithm based on nonsubsampled contourlet transform and pulse coupled neural netwrork”. Computer Applications, Vol.28 ,no.12,pp.164-167, 2008.

[7] Xiaobo Qu, Jingwen YAN, Hongzhi XIAO, Ziqian ZHU, “Image Fusion Algorithm Based on Spatial Frequency Motivated Pulse Coupled Neural Networks in Nonsubsampled Contourlet Transform Domain,”Acta Automatica Sinica, vol. 34, no.12, pp. 1508-1514, 2008

[8]Jiexiong Wang, Xiangyang Qi, XieWei Li, "Edge and local energy NSCT based remote sensing image fusion, Journal of the Graduate School of the Chinese Academy of Sciences”, vol.26, no.5, pp. 657-661, 2009.

[9]Qu Guihong, Zhang Dali, Yan Pingfan, “Information measure for performance of image fusion”. Electronics Letters, vol. 38, no.7, pp. 313-15, 2002.

[10]Xydeas, C.S.,” Petrovic, V.,Objective image fusion performance measure”, Electronics Letters, vol.36, no.3, pp. 308- 309, 2000. 